Classification

Physics Abstracts

61.30J - 61.16D

\title{
Ripple wavelength, amplitude, and configuration in lyotropic liquid crystals as a function of effective headgroup size
}

\author{
J. A. N. Zasadzinski $\left({ }^{*, 1}\right)$ and M. B. Schneider $(* *, 2)$ \\ (*) AT \& T Bell Laboratories, Murray Hill, New Jersey 07974, U.S.A. \\ $\left.{ }^{* *}\right)$ Department of Physics, Cornell University, Ithaca, New York 14853, U.S.A.
}

(Reçu le 10 février 1987, révisé le 2 juin 1987, accepté le 26 juin 1987)

\begin{abstract}
Résumé. - Nous présentons des mesures d'analyse thermique différentielle et des images obtenues au microscope électronique par cryofracture d'un lipide biologique. Il s'agit de la phase $\mathrm{P}_{\beta^{\prime}}$ de la dimyristoylphosphatidylcholine (DMPC) dopée par la dimyristoylphosphatidylethanolamine (DMPE*) ; les objets étudiés sont des liposomes multilamellaires en présence d'un excès d'eau. La DMPE* est utilisée comme dopant afin de compenser l'écart entre les aires de têtes polaires et de chaînes aliphatiques de la DMPC. Les résultats d'ATD montrent que la phase $P_{\beta^{\prime}}$ disparait pour une fraction molaire de 0,15 en DMPE*. Pour toutes les fractions molaires en DMPE* qui donnent une phase $\mathbf{P}_{\beta^{\prime}}$, nous observons deux types d'ondulations : une ondulation asymétrique de longueur d'onde $12,0 \mathrm{~nm}$ et d'amplitude inférieure à $6 \mathrm{~nm}$, et une ondulation symétrique de longueur d'onde incommensurable $22,0 \mathrm{~nm}$ et d'amplitude supérieure à $9 \mathrm{~nm}$. La présence de

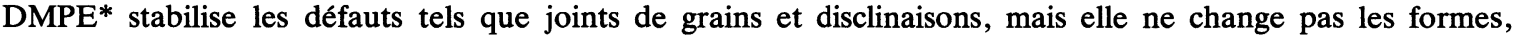
longueurs d'onde et amplitudes des ondulations. La DMPE* semble aussi empêcher l'annihilation des ondulations en dessous de la prétransition. Nous discutons ces résultats dans le cadre des modèles de continuum de la phase $\mathrm{P}_{\beta^{\prime}}$.
\end{abstract}

Abstract. - We present differential scanning calorimetry (DSC) and freeze-fracture electron micrographs of the $P_{\beta^{\prime}}$ phase of dimyristoylphosphatidylcholine (DMPC) multilamellar liposomes in excess water with varying fractions of N-methyl substituted dimyristoylphosphatidylethanolamine (DMPE*). DMPE* was chosen as a dopant to eliminate the mismatch between the areas of the polar headgroup and the hydrocarbon chains of DMPC. DSC results show that the $\mathrm{P}_{\beta^{\prime}}$ phase disappears at a DMPE* mole fraction of about 0.15 . Two distinct ripple configurations are observed at all DMPE* fractions that exhibited a $\mathbf{P}_{\boldsymbol{\beta}^{\prime}}$ phase ; an asymmetric ripple of wavelength $12.0 \mathrm{~nm}$ and amplitude less than $6 \mathrm{~nm}$ and a symmetric ripple of incommensurate wavelength $22.0 \mathrm{~nm}$ and an amplitude of at least $9 \mathrm{~nm}$. Defects, such as disclinations and grain boundaries are stabilized by DMPE*, but the ripple shapes, wavelength and amplitudes are unaffected. DMPE* also seems to prevent the annihilation of the ripples below the pretransition. The results are discussed in relation to continuum descriptions of the $\mathrm{P}_{\boldsymbol{\beta}^{\prime}}$ phase.

\section{Introduction.}

Phospholipids associate in the presence of water to form a variety of lyotropic liquid crystalline phases [1]. The most common is the lamellar phase which consists of symmetric bilayers intercalated with water. A wide range of physicochemical techniques have shown that phospholipid bilayers undergo

( $\left.{ }^{1}\right)$ Present address : Department of Chemical and Nuclear Engineering, University of California, Santa Barbara, California 93106, U.S.A.

${ }^{(2)}$ Present address: Lawrence Livermore National Lab, P.O. Box 808, Livermore, California 94550, U.S.A. temperature dependent, reversible, intralayer orderdisorder transitions $[2,3]$. In excess water, above the gel or main phase transition temperature, many phospholipids form an $\mathrm{L}_{\alpha}$ phase, a smectic A structure in which the intermolecular order in each half of the bilayer is short range and liquid-like and the intramolecular trans-gauche rotational order of the hydrocarbon chains is low [1-7].

As the temperature is lowered, some saturated phosphatidylcholines, notably dimyristoylphosphatidylcholine (DMPC) (Fig. 1a) and dipalmitoylphosphatidylcholine (DPPC) undergo a first order transition [4] to the $P_{\beta^{\prime}}$ phase. In this phase, the 
a)

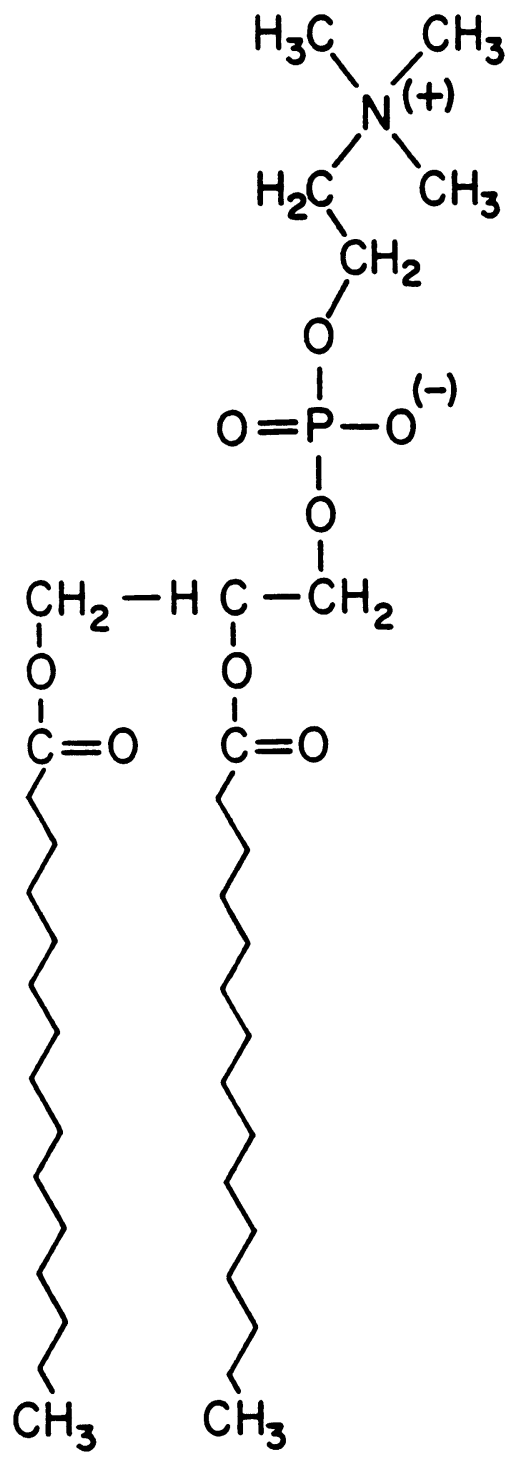

b)

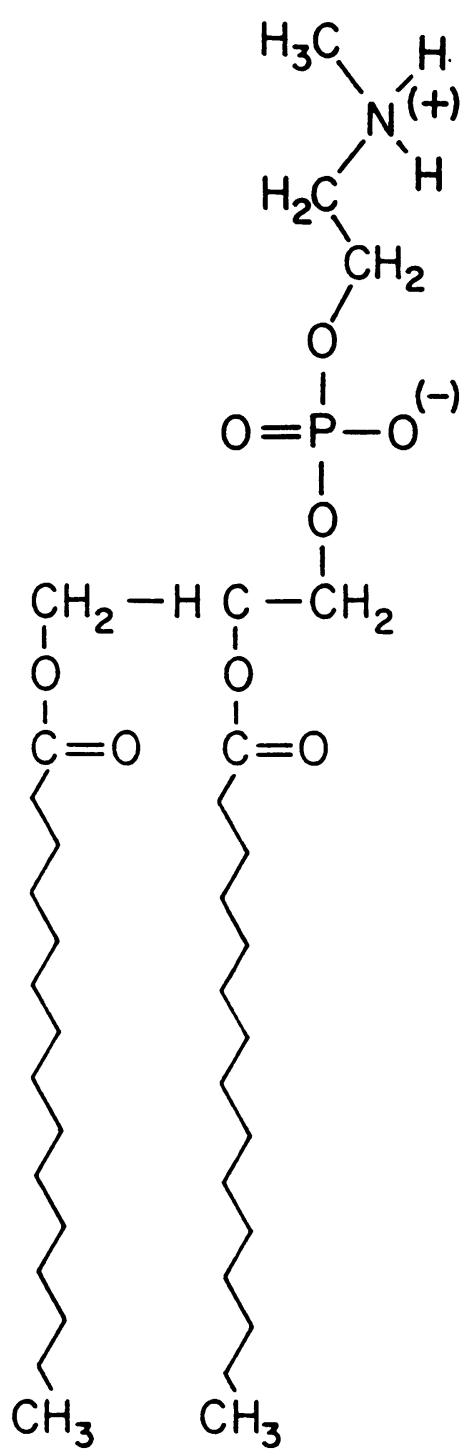

Fig. 1. - Schematic diagram of a) DMPC and b) DMPE*.

hydrocarbon chains are frozen into a nearly all trans configuration $[1,5]$ and are packed in a two-dimensional hexagonal lattice $[1,4,20]$, the intralayer selfdiffusion coefficient is small [16]. There still exists some disagreement as to whether the molecules are tilted $[1,4]$ or perpendicular to the bilayer [21]. The $\mathbf{P}_{\beta^{\prime}}$ phase is marked by regular intrabilayer corrugations ; hence the common name of ripple phase. X-ray diffraction $[1,2,4,7,24]$ and freeze-fracture electron microscopy $[6,18,20,22]$ find the ripple wavelength of DMPC in excess water to be $12.0 \mathrm{~nm}$. Freeze-fracture images also present regions of second ripple shape with a wavelength of about $23.0 \mathrm{~nm}$ $[6,18]$, although these longer wavelength ripples have not been reported in X-ray diffraction. The structural details of the corrugations are undetermined ; some models predict a sinusoidal modulation $[7,8,11]$ while others predict a sawtooth modulation $[1,6,9,20,24]$. The reported ripple amplitudes range from $8 \mathrm{~A}$ [4] to $25 \mathrm{~A} \mathrm{[7].}$
If the temperature is lowered further, there is a second phase transition, called the pretransition, to the $L_{\beta^{\prime}}$ phase. In this phase, the bilayers are again flat lamellae and the hydrocarbon chains are fully extended (all trans configuration) and tilted with respect to the bilayer normal [1,4]. Within the bilayer, the hydrocarbon chains are packed in a rhombic lattice [1,4]. This phase is similar to a smectic G.

Other saturated phospholipids such as those with the phosphatidylethanolamine headgroup, go directly from the $\mathrm{L}_{\alpha}$ phase to the $\mathrm{L}_{\beta}$ phase, at the chain freezing temperature [13]. The $\mathrm{L}_{\beta}$ phase, similar to a smectic $B$, differs from the $L_{\beta^{\prime}}$ phase in that the chains are perpendicular to the bilayer normal and are packed into a hexagonal lattice within the layers $[1-3,5]$.

In addition to their biological significance, these phases have attracted significant interest because of their two-dimensional intramolecular order and be- 
cause of the unusual occurrence, as a function of temperature, of a phase of higher symmetry $\left(\mathrm{P}_{\beta^{\prime}}\right)$ between two phases of lower symmetry $\left(\mathrm{L}_{\alpha}\right.$ and $\mathrm{L}_{\beta^{\prime}}$ ).

The physics underlying the pretransition and the ripple configuration of saturated phosphatidylcholines is poorly understood. Many theories of the ripple phase have suggested that the ripples in the $P_{\beta^{\prime}}$ phase are caused, at least in part, by the mismatch between the area projected in the bilayer plane by the hydrocarbon chains and the polar headgroup [8-11, 23]. For saturated phosphatidylcholines (PC), the headgroup is relatively large compared to the hydrocarbon chains [12] at temperatures below the main transition. In contrast, the projected size of the phosphatidylethanolamine (PE) headgroup is similar to that of the hydrocarbon chains [12]. In these theories, the chains are tilted in the PC's $L_{\beta^{\prime}}$ phase, but not in the PE's $L_{\beta}$ phase in order to accommodate the area mismatch of the former. The rippling in the PC's $P_{\beta^{\prime}}$ phase is believed to be an alternate structural way of satisfying the area incompatibility.

In this study, differential scanning calorimetry (DSC) and freeze-fracture electron microscopy were used to examine DMPC bilayers modified by doping with varying fractions of $\mathrm{N}$-methyl substituted dimyristoylphosphatidylethanolamine (DMPE*). In DMPE*, two of the PC's choline methyl groups are replaced by hydrogen, making the effective size of the headgroup smaller (Fig. 1b). The PE headgroup differs from the $\mathrm{PC}$ headgroup in that all three of the choline methyl groups are replaced by hydrogen. The phase diagram of DMPE*, like that of DMPE, does not exhibit a $P_{\beta^{\prime}}$ phase. The main transition temperatures of DMPE and its methylated derivatives are higher that those of DMPC, increasing proportionately to the number of hydrogens substituted for methyl groups [13]. This suggests that in addition to the difference in headgroup size between $\mathrm{PC}$ and $\mathrm{PE}$, the binding between headgroups is stronger in DMPE and its methylated derivatives than in DMPC. For DMPE*, the main transition is about $18^{\circ} \mathrm{C}$ higher than in pure DMPC [13]. The smaller steric effects in DMPE* allows the ammonium hydrogen to approach the phosphate more closely than can any of the methyl hydrogens of the choline group (Fig. 1). Both the inter- and intramolecular association is thereby increased [14].

In our study, the fraction of DMPE* in the $P_{\beta^{\prime}}$ phase is less than 0.15. Each DMPE* molecule is, on average, surrounded by DMPC molecules ; the strength of the headgroup interactions was close to that of pure DMPC. The primary effect of the DMPE* $^{*}$ is to decrease the average headgroup size. The main transition temperature at 25 mole \% DMPE*-75 mole \% DMPC was $27.5^{\circ} \mathrm{C}$ compared to $25^{\circ} \mathrm{C}$ for pure DMPC, and $43{ }^{\circ} \mathrm{C}$ for pure DMPE*, suggesting that the headgroup association was dominated by DMPC.

\section{Materials and methods.}

Stock solutions of $50 \mathrm{mg} / \mathrm{ml}$ DMPC (Avanti Polar Lipids ; Birmhingham, Alabama, 35216) and $50 \mathrm{mg} / \mathrm{ml}$ DMPE* (Calbiochem-Behring Corp., La Jolla, California 92307) in 2:1 vol/vol chloroformmethanol solution were mixed in the appropriate quantities in glass vials to form mixtures of 0.5 100 mole \% DMPE*. The solvent was removed by rotary evaporation at $40^{\circ} \mathrm{C}$. Doubly-distilled, deionized water was added to the dried lipids to give a final concentration of $5 \mathrm{wt} \%$ lipid. The lipids were gently shaken, then kept at a temperature of $44^{\circ} \mathrm{C}$ for several hours. Under these conditions, PC's and PE's are known to form micron sized, multilamellar liposomes.

Differential scanning calorimetry (DSC) was performed in a Perkin-Elmer DSC-4 cooled by a Perkin Elmer Intracooler (South Pasadena, California 91030). A droplet containing $3 \mathrm{mg}$ lipid was sealed in a stainless steel sample pan with an O-ring seal. Endotherms were recorded at a heating rate of $5^{\circ} \mathrm{C} / \mathrm{min}$. An average over several cycles was taken. Endotherms were reproducible to $\pm 0.3^{\circ} \mathrm{C}$.

Samples for electron microscopy were prepared by sandwiching a small droplet $(0.1-0.5 \mu \mathrm{l})$ of the lipid-water mixture between two copper planchettes (Balzers BUO-12-056T ; Hudson, New Hampshire) to form a $10-50 \mu \mathrm{m}$ thick layer. Previously, the copper planchettes had been etched for $1 \mathrm{~s}$ in concentrated nitric acid to remove any contamination and roughen the planchettes' surface. The apparatus used for rapid freezing was a modified version of a Balzers Cryojet QFD 020 jet-freeze device. The sample sandwiches were mounted on a teflon support arm and placed in a temperature controlled oven located vertically over the jets of the Balzers Cryojet. Sample temperature was adjusted to be in the middle of the range of the $\mathrm{P}_{\beta^{\prime}}$ phase as measured by DSC. Temperature control prior to freezing was better than $0.1{ }^{\circ} \mathrm{C}$. The samples were equilibrated at the appropriate temperature for a few minutes before a solenoid-actuated mechanism opened a teflon door at the bottom of the oven and dropped the teflon arm and samples between the opposed jets of the Balzers cyrojet. A photodiode switch initiated the high velocity flow of liquid propane, cooled by liquid nitrogen to $-180^{\circ} \mathrm{C}$, which impinged on the copper sandwich from both sides to provide a minimum cooling rate of $10,000^{\circ} \mathrm{C} / \mathrm{s}$. Less than $0.1 \mathrm{~s}$ elapsed between the time the sample left the oven and it was hit by the liquid propane jet. The frozen copper sandwiches were stored under liquid nitrogen until transferred into a spring-loaded «mousetrap » carrier. The 
loaded sample carrier was mounted onto the liquid nitrogen-cooled coldfinger in a Balzers 400 freezeetch device and the vacuum chamber was evacuated to better than $10^{-7}$ torr. The temperature of the sample carrier and coldfinger were adjusted to $-170{ }^{\circ} \mathrm{C}$ and the spring mechanism was externally actuated, thereby fracturing the samples. The fracture surfaces were immediately replicated by evaporating $1.5 \mathrm{~nm}$ of a platinum carbon mixture from an electrode at a $45^{\circ}$ angle to the fracture surface, followed by a $15 \mathrm{~nm}$ thick film of carbon at normal incidence to increase the mechanical stability of the replica. The samples and replicas were removed from the vacuum chamber and the samples and copper planchettes were dissolved in chromic acid, leaving the platinum-carbon replicas behind. The replicas were washed in a $50 \% \mathrm{vol} / \mathrm{vol}$ chloroethanol-water mixture, rinsed in doubly-distilled water, and collected on formvar-coated 50 mesh gold electron microscope grids (Pelco, Tustin, California). The replicas were examined in a JEOL 100B scanning transmission electron microscope in the conventional transmission mode using $80 \mathrm{kV}$ electrons. The side entry goniometer of the JEOL 100B was adjusted so that the specimen was oriented perpendicular to the electron beam (within $1^{\circ}$ ). Images were recorded on Kodak electron image plates. Shadows (absence of platinum) appear light in the prints.

Ripple periodicities were measured using optical diffraction from the electron microscope negatives [15]. A collimated and spatially filtered helium-neon laser beam was spread to about $1 \mathrm{~cm}$ diameter and passed through a selected area of the negative. After passing the beam through a weakly converging lens, the diffraction pattern of the negative was recorded on Polaroid film. Absolute spacings were calculated by comparison with diffraction patterns of equal magnification TEM images of catalase crystals (SPI, West Chester, Penn.) of known d-spacing. Areas chosen for optical diffraction were from large, planar liposomes with well-aligned ripples. Ripple periodicities were measured only from those areas that exhibited at least two orders of reflection, indicating well aligned, parallel ripples (see Fig. 3). Periodicities were accurate to within $\pm 0.5 \mathrm{~nm}$.

\section{Experimental observations.}

3.1 DIFFERENTIAL SCANNING CALORIMETRY. The same liposome solutions were used for the DSC scans and the freeze-fracture experiments to allow for direct comparison between the two techniques. The solution is rather dilute for DSC, so only low sensitivity scans, at a heating rate of $5{ }^{\circ} \mathrm{C} / \mathrm{min}$, were performed. Typical DSC traces are shown in figure 2. For DMPE* mole fractions up to 0.1 , a distinct pretransition $(\mathrm{P})$ is visible as a small, broad

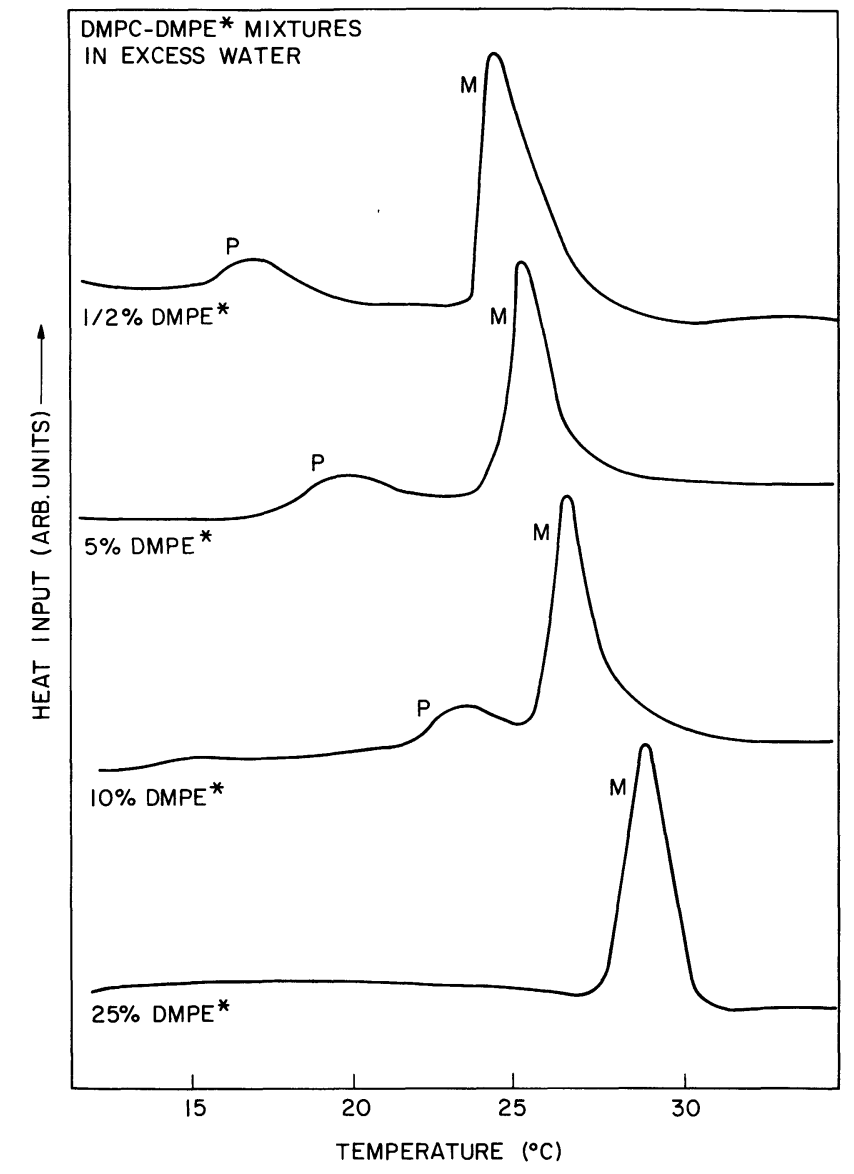

Fig. 2. - Typical DSC traces at heating rates of $5^{\circ} \mathrm{C} / \mathrm{min}$ for different mixtures of DMPE* and DMPC. P marks the pretransition and $\mathrm{M}$ the main transition. The scans at $0 \%$,

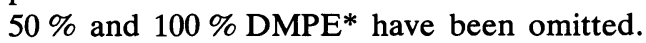

peak at $3-10{ }^{\circ} \mathrm{C}$ below the main transition (M). For $\mathrm{DMPE}^{*}$ mole fractions of 0.25 and above, the pretransition exotherm $(\mathrm{P})$ disappears and only the main transition exotherm (M) remains. The onset temperature of the main transition of pure DMPC was $24.4 \pm 0.3{ }^{\circ} \mathrm{C}$ in good agreement with published values [4]. The onset of the pretransition of pure DMPC was $14.6 \pm 0.3^{\circ} \mathrm{C}$. The main transition of DMPE $^{*}$ was $43.5 \pm 0.3^{\circ} \mathrm{C}$, also in good agreement with published values [13]. (The onset temperature of a transition is the intersection of the tangent to the leading edge of the DSC peak with the baseline.) The DSC experiments did not have sufficient resolution to determine the temperature at which the phase transition was completed, so the width of the two phase regions could not be determined. The onset temperatures of the main transition appear to be linear in $\mathrm{DMPE}^{*}$ fraction, suggesting that the mixing of the two components is close to ideal. The main phase transition temperature onset only increases by about $2-3{ }^{\circ} \mathrm{C}$ from 0 to 25 mole \% DMPE*, suggesting that the head group interactions are still DMPC-like. The pretransition 
temperature onset also increases linearly with increasing DMPE* mole fraction, but with a much greater slope. Extrapolating, the $\mathrm{P}_{\beta^{\prime}}$ phase disappears at a DMPE* mole fraction of about 0.15 . These results show that the primary effect of $\mathrm{DMPE}^{*}$ is to eliminate the mismatch between the headgroups and the hydrocarbon chain areas, thereby eliminating the need for $\mathbf{a}_{\boldsymbol{\beta}^{\prime}}$ phase. This is not to say that DMPE* does not preferentially locate in regions of large headgroup compression in the ripples as has been suggested $[6,16,17]$. The DSC results do show that DMPE* does not segregate to a «macroscopic» separate phase but remains intimately mixed with DMPC.

3.2 ELECTRON MiCROSCOPY. - Liposomes of all mole fractions that show a pretransition exhibit two characteristic ripple structures when quenched from between the pre- and main transitions. The smaller wavelength ripple, called the $\Lambda / 2$ phase by Ruppel and Sackmann [6], is an asymmetric modulation of wavelength $12.0 \mathrm{~nm}$ (Fig. 3a). The ripples are usually in phase between layers. It is difficult to determine the amplitude of the ripples from freeze-fracture images unambiguously [18], but evaluation of the shadows in the platinum-carbon film show that the peak-to-trough height is substantially less than $6.0 \mathrm{~nm}$, or half the wavelength. Figure $4 \mathrm{a}$ shows that if the height of a symmetric ripple were greater that $60 \AA$, more than half of the ripple would be uncoated by platinum and would appear light in the print. In general, the angle of inclination, $\vartheta_{2}$, on the back side of the ripple must be less than or equal to $\vartheta$, the shadowing angle, $\left(45^{\circ}\right.$ in these experiments) in order for the unidirectional evaporating platinum to strike the back side of the ripple. If $\vartheta_{2}$ was less than $45^{\circ}$, then both sides of the ripples would be coated with metal, and the maximum height of the ripple would be $6.0 \mathrm{~nm}$. Figure $4 \mathrm{~b}$ illustrates this for an asymmetric ripple. A close inspection of the ripples in figure $3 a$ shows that the bulk of the ripple is coated by metal, hence the amplitude of the ripple is less than $60 \AA$. It can also be shown that the ripples are asymmetric, as they appear different depending on their relative orientation with respect to the shadow direction. The ripples have two characteristic shadow patterns, a medium gray with white borders, labelled $\mathrm{A}$ in figure $3 \mathrm{a}$, and a lighter grey with a dark grey border at $\mathrm{B}$. This is consistent with the asymmetric sawtooth drawn in figure $4 \mathrm{~b}$.

Often, in other liposomes, or even in other areas of the same liposome, a second ripple structure is observed. The wavelength is $22.0 \mathrm{~nm}$, about 1.8 times the wavelength of the shorter ripples and incommensurate with them. These $\Lambda$ phase ripples, as denoted by Ruppel and Sackmann [6], are symmetric and appear identical regardless of the shadow direction. They appear to have $\mathrm{a} \ll \mathrm{W} »$ shape; $\mathrm{a}$
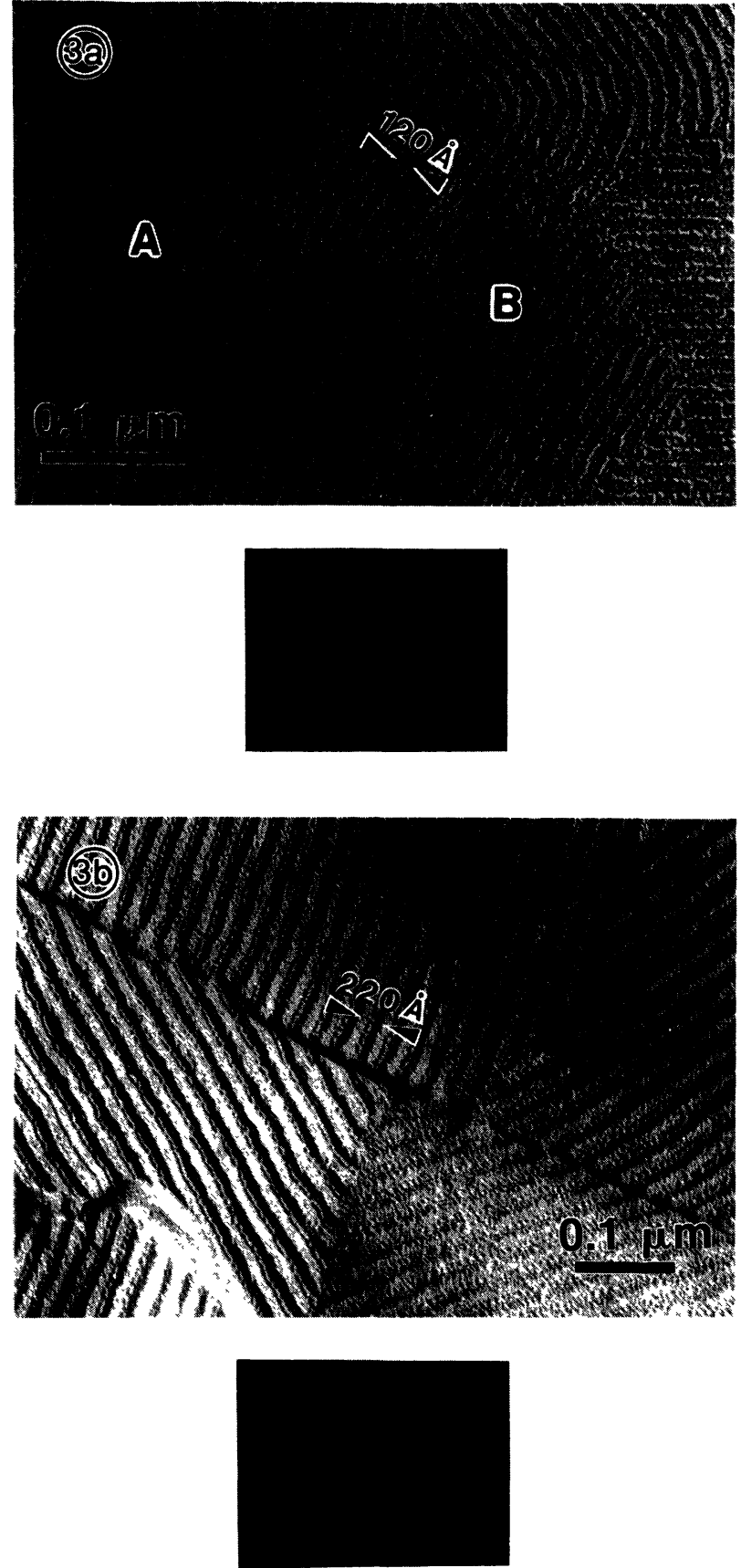

Fig. 3. - Typical freeze-fracture electron micrograph images of liposomes quenched from the $\mathrm{P}_{\beta^{\prime}}$ phase. In (a), are $\Lambda / 2$ phase ripples. $A$ and $B$ mark ripples of different shadowing showing that the ripples are asymmetric (see Fig. 4b). Optical diffraction from negatives is below and shows two sharp reflections with a period of $12.0 \mathrm{~nm}$. In (b) are $\Lambda$ phase ripples. Optical diffraction from negative shows four sharp reflections with a period of $22.0 \mathrm{~nm}$.

small extra peak in the centre of a deep trough. One side of the large part of each ripple appears unshadowed, so it must have a slope of at least $45^{\circ}$. The width of the large part of the ripple is about $18.0 \mathrm{~nm}$, so the ripple amplitude is at least $9.0 \mathrm{~nm}$. One side of the short, centre ripple of width $4.0 \mathrm{~nm}$ is also 


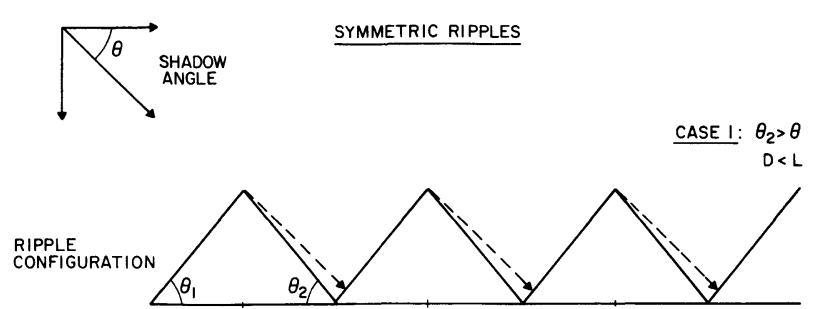

SHADOW
PATTERN
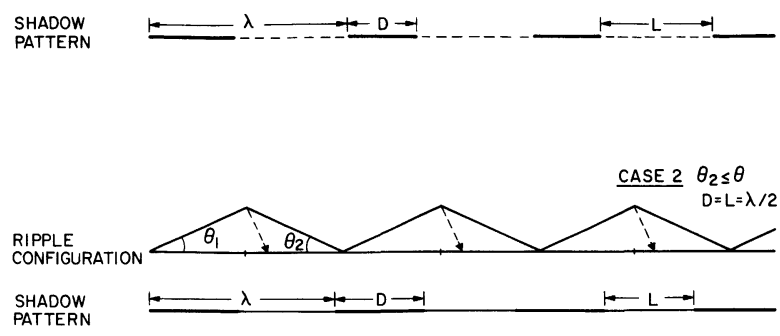

Fig. 4a. - Predicted ripple shadow patterns for symmetric ripples if $\vartheta>\vartheta_{2}$, most of the ripple is uncoated by platinum and will appear grey-white in micrographs. If $\vartheta \leqslant \vartheta_{2}$ all the ripple will be coated and will appear dark grey-light grey in micrographs.

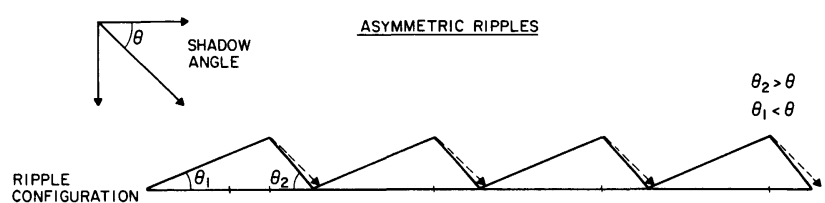
$\underset{\substack{\text { SHADOW } \\ \text { PATTERN }}}{\text {. }}$

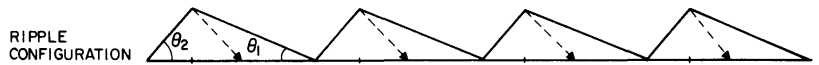

SHADOW
PATTERN

Fig. 4b. - Asymmetric ripples will appear different depending on its relative orientation with respect to the shadow direction. Grey-white if the long side is oriented towards the shadow direction, dark grey-light grey if away. Compare to the shadow patterns at areas A and B in figure $3 a$.

unshadowed ; the amplitude of this centre portion is at least $2.0 \mathrm{~nm}$ (see Fig. 4a). These $\Lambda$ phase ripples are believed to be generated from the $\Lambda / 2$ phase by rotating every other $\Lambda / 2$ ripple by $180^{\circ}$ and then tilting each half in opposite directions [6]. As the $\Lambda$ and $\Lambda / 2$ phases have both incommensurate wavelengths of $22.0 \mathrm{~nm}$ and $12.0 \mathrm{~nm}$, a tilt of the $\Lambda / 2$ ripple by $\pm 23.6^{\circ}$ is necessary. Combining two $\Lambda / 2$ ripples in this manner would show up in a freeze-fracture replica as either one short part or one long part of the combined ripple being unshadowed. However, both a short part and a long part of the $\Lambda$ ripple is unshadowed. This raises the possibility that the $\Lambda$ is a second configuration entirely. Often, the $\Lambda$ phase ripples are not in phase between layers (See Fig. 3b).

3.3 EFFECTS OF DMPE* ON RIPPLE CONFIGURATION. - Figures $5 \mathrm{a}$ and $5 \mathrm{~b}$ are liposomes of 0.5 mole \% DMPE* quenched from $20^{\circ} \mathrm{C}$ in the $\mathrm{P}_{\boldsymbol{\beta}^{\prime}}$ phase. The liposomes are predominantly $\Lambda / 2$ phase ripples of wavelength $120 \pm 5 \mathrm{~A}$. The ripples are straight for distances of up to a few microns and

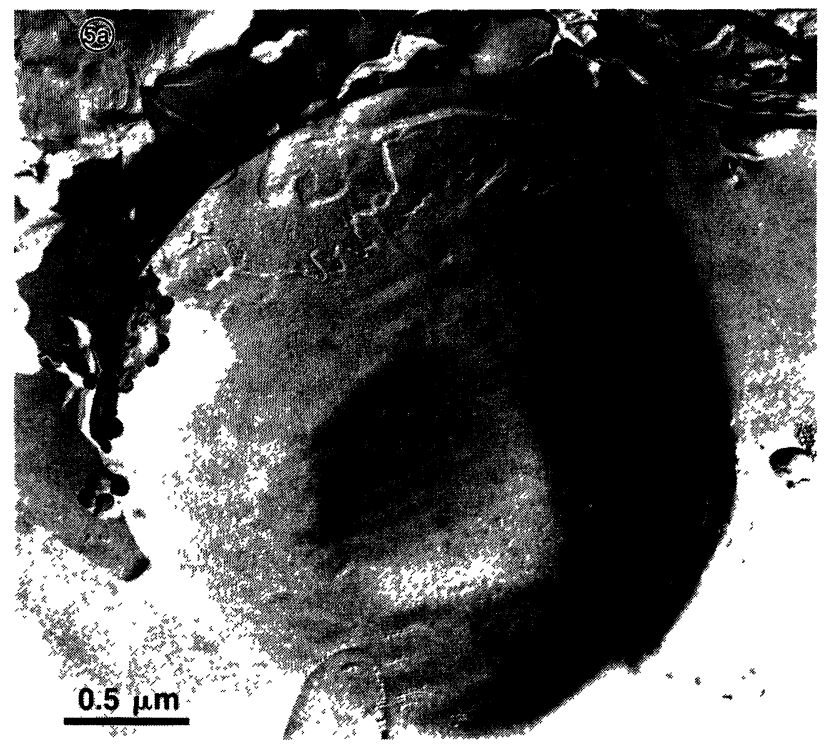

Fig. 5a. - Large liposome of 0.5 mole \% DMPE* quenched from the $\mathrm{P}_{\beta^{\prime}}$ phase. Only $\Lambda / 2$ phase ripples are present.

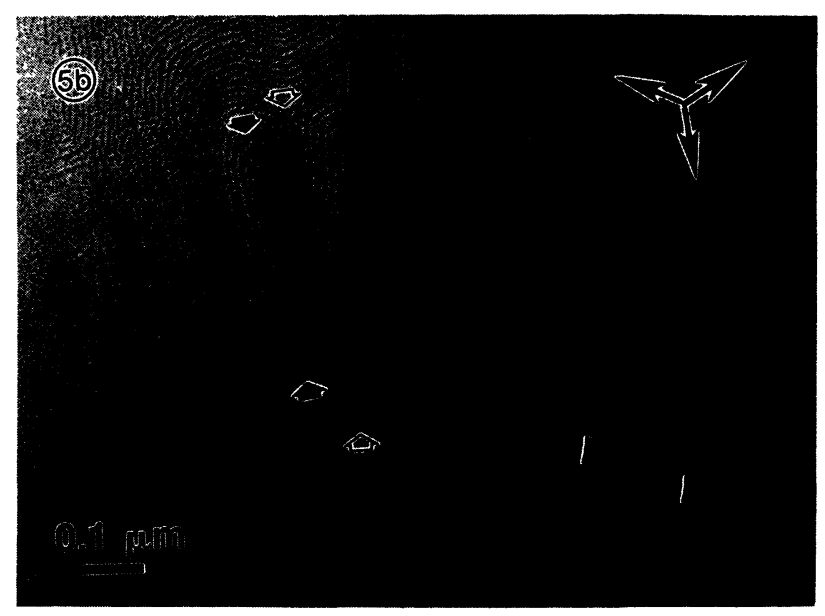

Fig. 5b. - Array of $+1,-1$ disclinations in $\Lambda / 2$ ripples, dissociated into half-integer disclinations in 0.5 mole \% DMPE* liposome. Three-fold symmetry in ripple directions is evident. Broad filled arrows mark $+1 / 2$ disclinations and open arrows mark the $-1 / 2$ disclinations. The array forms a macro-ripple in the liposome surface. Small filled arrows mark two $\Lambda / 2$ phase ripples joined short side to short side at centre of defect. 
seem to follow the lattice directions. A three-fold symmetry can be seen in figure $5 \mathrm{~b}$; all the ripples in the photograph traverse in one of three directions, separated by $120^{\circ}$. As the hydrocarbon chain packing in the $P_{\beta^{\prime}}$ phase is hexagonal [4], the ripple orientation is likely correlated with the hydrocarbon chain lattice.

Defects are common in the ripple arrangement at all DMPE* concentrations. Integer strength disclinations are observed in the $\Lambda / 2$ phase ripples, [6] which are decomposed into quasi-half strength disclinations connected by line defects. An array of +1 , - 1 disclinations dissociated into half integer disclinations joined by defect lines is visible in figure $5 \mathrm{~b}$. The open arrows mark the $-1 / 2$ disclinations and the filled arrows the $+1 / 2$ disclinations. They form a « macro-ripple » in the liposome surface. The defect line joining each half disclination appears to be two $\Lambda / 2$ ripples joined short side to short side, giving the pattern shown at the small arrows. Although this shadow pattern is similar to that of the $\Lambda$ phase ripples (see Fig. 3b), little of the ripple is uncoated by platinum as opposed to the $\Lambda$ phase ripple in figure $3 b$, in which more than half the ripple is uncoated.

The $\Lambda$ phase is absent except for a few, smaller liposomes such as the one in figure 5c. The threefold symmetry present in the $\Lambda / 2$ phase is also present in the $\Lambda$ phase. The defect topology of the $\Lambda$ phase can accommodate highly curved small liposomes better than the $\Lambda / 2$ phase [6]. Half-integer disclinations are topologically allowed in the symmetric $\Lambda$ phase, but only integer strength disclinations are allowed in the asymmetric $\Lambda / 2$ phase.

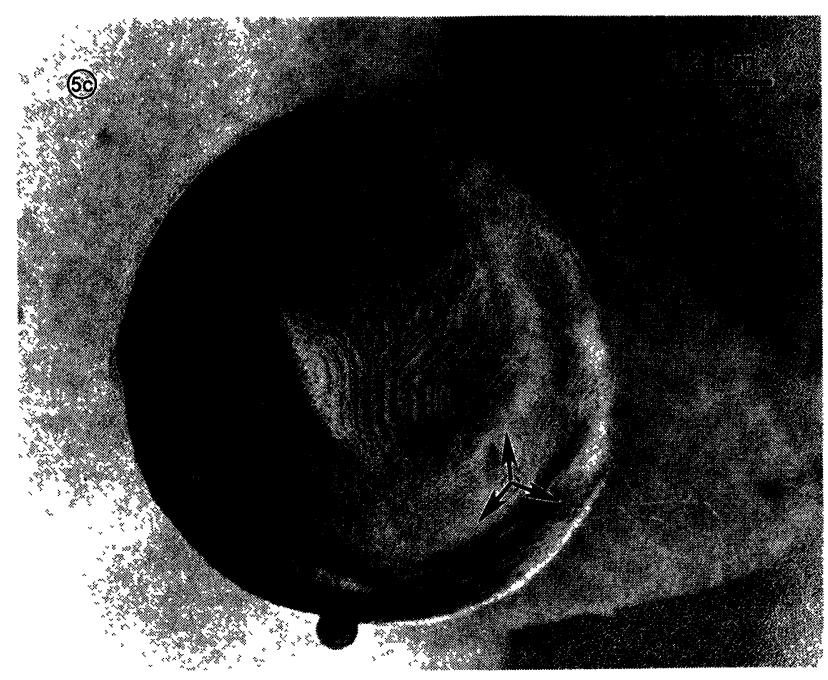

Fig. 5c. - Smaller 0.5 mole DMPE* liposome of about $0.5 \mu \mathrm{m}$ diameter exhibiting $\Lambda$ phase ripples, again with three-fold directional symmetry. $\Lambda$ phase ripples are more common on smaller particles because of defect topology (see text).
Harris [19] showed that to cover a spherical surface with a regular pattern, surface disclinations were required and their net strengths must sum to 2 .

When the fraction of DMPE* was increased to 2 mole $\%$, the fraction of $\Lambda$ phase ripples also increased. In many liposomes, both ripple configurations exist simultaneously (see Fig. 6a, 6b). The ripple wavelengths are unchanged, $12.0 \mathrm{~nm}$ for the $\Lambda / 2$ phase (B in Fig. 6a) and $22.0 \mathrm{~nm}$ for the $\Lambda$ phase (A in Fig. 6a). In the smaller liposomes, as in

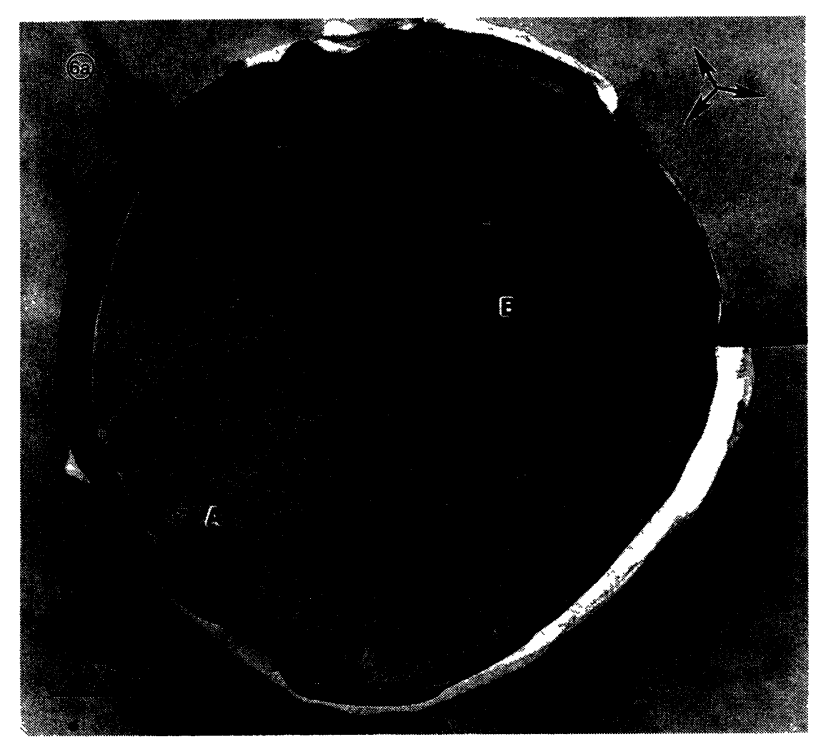

Fig. 6a. -2 mole \% DMPE* liposome with both $\Lambda$ phase (A) and $\Lambda / 2$ phase ripples. Ripple wavelengths and configurations are unchanged.

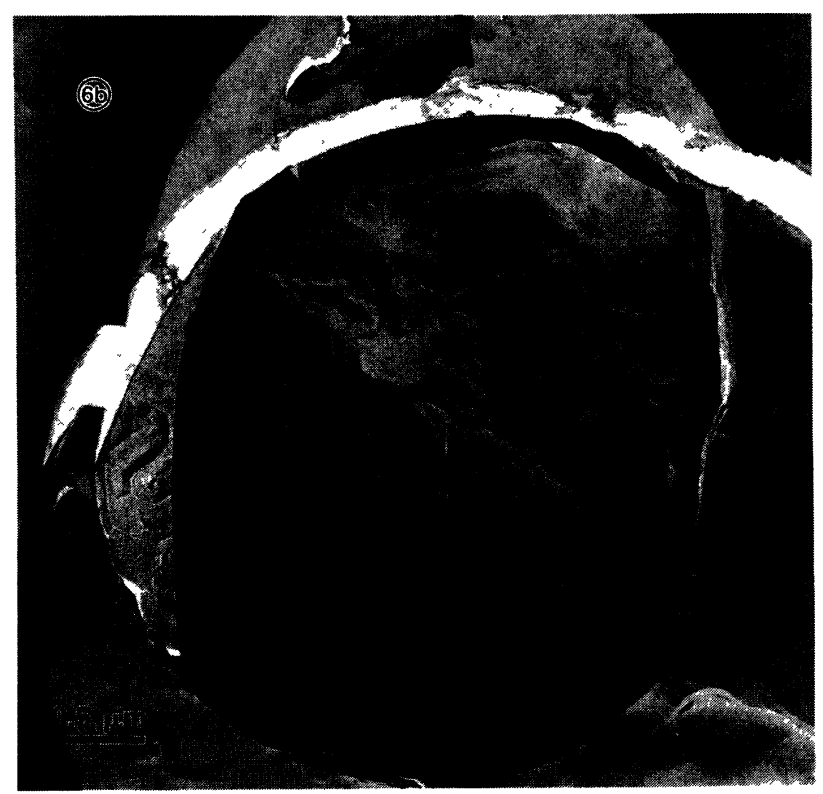

Fig. 6b. - Large macro-ripples form around disclinations in the $\Lambda / 2$ phase in certain 2 mole \% DMPE* liposomes, although not in areas of $\Lambda$ phase. 
the case of the 0.5 mole $\% \mathrm{DMPE}^{*}$ liposomes, the $\Lambda$ phase predominates. Compared to the 0.5 mole $\%$ liposomes, the ripples in the 2 mole \% DMPE* liposomes appear to be straight for shorter distances and there appears to be many more defects. Large macro-ripples form around disclinations in the $\Lambda / 2$ phase in some liposomes (Fig. 6b). These macro-ripples may be related to the defect topology of the $\Lambda / 2$ phase.

Images of 5 mole \% DMPE* quenched from the $\mathrm{P}_{\beta^{\prime}}$ phase show significant fractions of both ripple configurations (Fig. 7a, 7b). Surprisingly, the $\Lambda / 2$ phase wavelength remains $12.0 \mathrm{~nm}$ and the $\Lambda$ phase wavelength remains $22.0 \mathrm{~nm}$. Again, the fraction of $\Lambda$ phase seems to increase with increasing DMPE* fraction, although it is difficult to quantify because of the limited number of micrographs taken, the

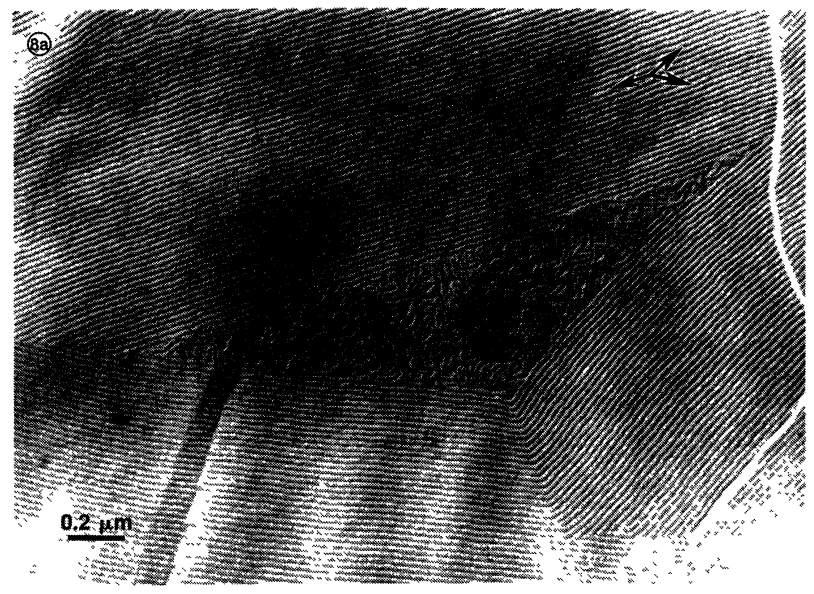

Fig. 8a. - 10 mole \% DMPE* liposome quenched from the $\mathrm{P}_{\beta}$, phase. Sharp, $30^{\circ}$ grain boundaries limit an area of jumbled $\Lambda$ ripples.
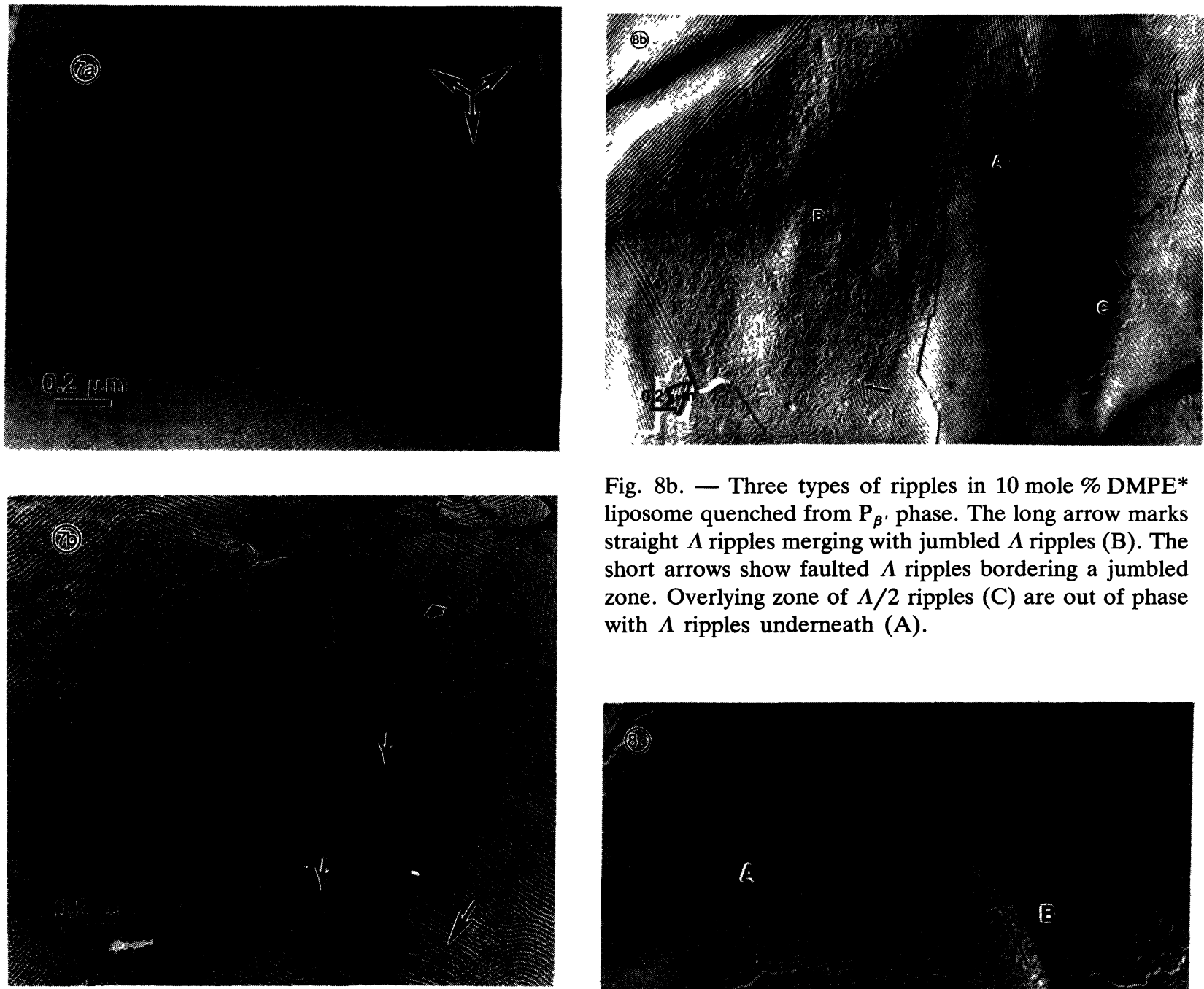

Fig. 8b. - Three types of ripples in 10 mole \% DMPE* liposome quenched from $\mathrm{P}_{\beta^{\prime}}$ phase. The long arrow marks straight $\Lambda$ ripples merging with jumbled $\Lambda$ ripples (B). The short arrows show faulted $\Lambda$ ripples bordering a jumbled zone. Overlying zone of $\Lambda / 2$ ripples (C) are out of phase with $\Lambda$ ripples underneath (A).

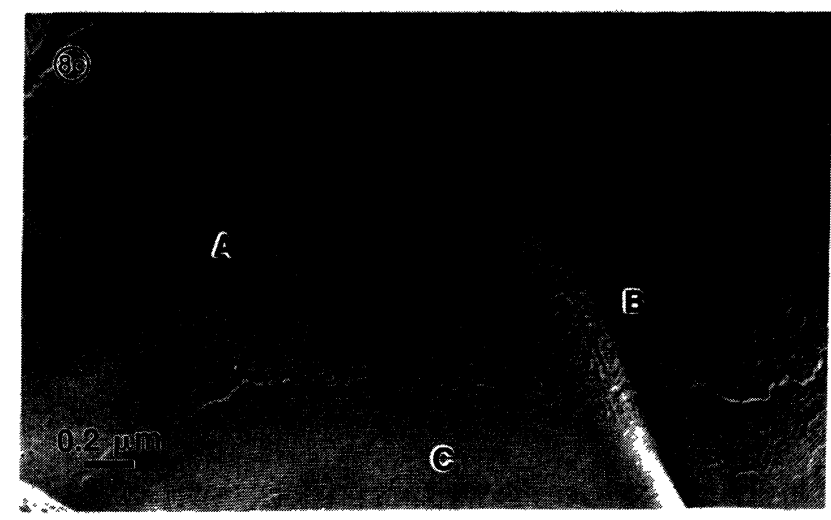

$\mathrm{P}_{\beta^{\prime}}$ phase. Significant fractions of both ripples are present. The three-fold symmetry is preserved. The short arrow in (b) marks a $60^{\circ}$ grain boundary, which is twice as sharp as a typical $120^{\circ}$ boundary. The medium arrows mark triangular patches and the long arrow a small region of $\Lambda$ phase.

Fig. 8c. $-\Lambda$ (A) and $\Lambda / 2$ (C) ripples bordering a triangular region of jumbled $\Lambda$ ripples (B). The jumbled area persists through several layers. 
unknown fracture mechanics of the ripples, etc.. The ripple configurations do not appear to change and the three-fold ripple directional symmetry remains. The number and type of defects increases as well. In figure $7 \mathrm{~b}$, the broad arrow marks a sharp-angle grain boundary of about $60^{\circ}$ instead of the usual $120^{\circ}$. There are small triangular patches at the medium arrows and a small patch of $\Lambda$ phase at the long arrow. DMPE* seems to stabilize grain boundaries between different ripple orientations as well as phase boundaries between $\Lambda / 2$ and $\Lambda$ patches.

Images of 10 mole \% liposomes quenched from the $\mathrm{P}_{\beta^{\prime}}$ phase exhibit both the $\Lambda / 2$ and $\Lambda$ phases, again with wavelengths of $12.0 \mathrm{~nm}$ and $22.0 \mathrm{~nm}$ respectively (see Fig. 8a-c). The number of defects and the fraction of the $\Lambda$ phase ripples have increased over smaller mole \% DMPE* samples. The diminished stability of the $P_{\beta^{\prime}}$ ripples might be the result of a decrease in the ripple translational order. In many of the 10 mole \% liposomes, both $\Lambda$ and $\Lambda / 2$ phases coexist with « jumbled » patches of $\Lambda$ like ripples (Fig. 8a-c). The jumbled patches are usually contained in triangular regions bordered by better ordered ripples. Often, the well-ordered ripples meet at sharp angles like the $30^{\circ}$ grain boundaries at the left and right of figure 8a. The jumbled ripples are $\Lambda$-like; they have the same shadow pattern and characteristic $W$ shape. At the long arrow in figure $8 \mathrm{~b}$, well ordered $\Lambda$ ripples can be seen to merge continuously with the jumbled ripples.

In figure $8 \mathrm{c}$, a patch of jumbled $\Lambda$ ripples (B) is bordered by both $\Lambda$ (A) and $\Lambda / 2$ ripples (C). The jumbled patch can be seen to persist over several bilayers. All three types of ripples are also observed in figure 8b. At the broad arrows, distorted $\Lambda$ phase ripples are present. They appear to be faulted and may have two small peaks per large trough instead of one. The $\Lambda / 2$ ripples at $\mathrm{C}$ are not in phase with the $\Lambda$ ripples in the layers above them; this is not surprising as they are incommensurate. At 50 mole \% DMPE*, the pretransition has disappeared and liposomes quenched from temperatures below the main transition are flat (Fig. 9).

Many authors have observed that contaminants such as DMPE* stabilize disclinations and other defects such as grain boundaries $[6,16,17]$. Impurities in the DMPC liposomes seem to locate preferentially in regions of large strain around defect cores and in areas of large curvature within the ripples [17]. It is not surprising that the smaller headgroup of DMPE* should locate in regions of higher headgroup compression, and thereby stabilize certain defects. This could explain why the number of defects increase with DMPE* mole fraction.

To explore this idea, a sample of 5 mole \% DMPE* was quenched from a temperature a few degrees below the pretransition. Figure 10a

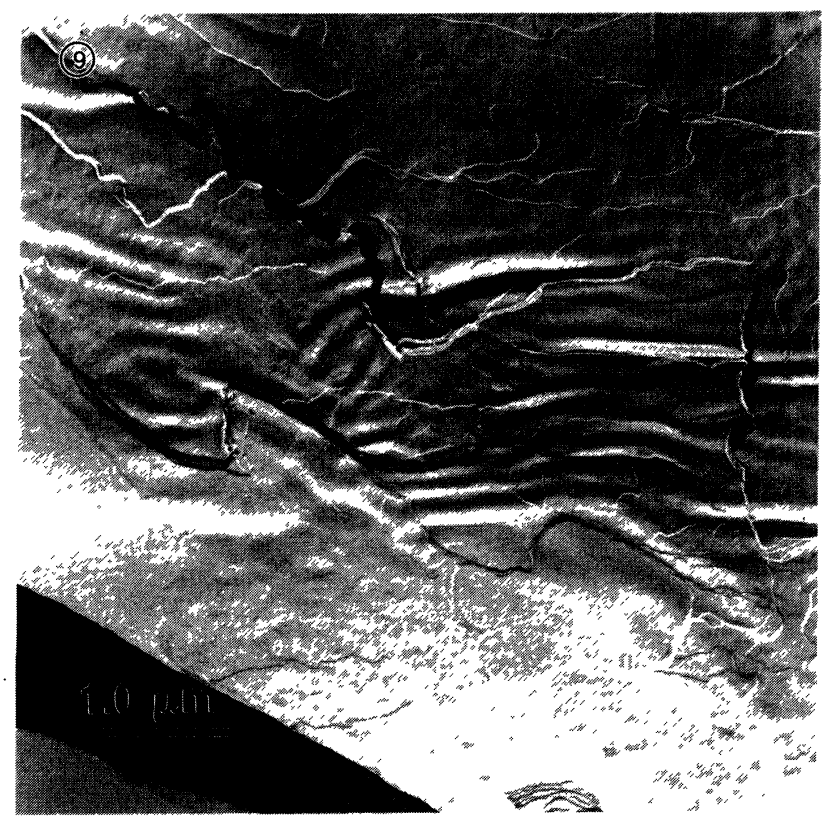

Fig. 9. - A 50 mole \% DMPE* liposome quenched from a few degrees below the main transition. The bilayers are flat and no ripples are seen.

shows one such liposome which was briefly cooled into the $L_{\beta^{\prime}}$ phase and then quenched. A new type of symmetric ripple is present with a wavelength of $24.0 \mathrm{~nm}$. The ripples have three-fold symmetry as shown in the higher magnification view in figure 10b. The ripples are arranged in a mosaic texture of patches of a few parallel ripples of a few tenths of a micron in length. At temperatures below the pretransition in pure DMPC liposomes, the bilayers are flat and the hydrocarbon chains are arranged in an orthorhombic lattice [4]. It appears that the DMPE* prevents the annihilation of the ripples, although the wavelength increases to about double that of the $\Lambda / 2$ phase. The DMPE* may also distort the orthorhombic lattice of the hydrocarbon chains in the $L_{\beta^{\prime}}$ phase because the angle between ripples is $120^{\circ}$, implying an underlying hexagonal arrangement. DMPC liposomes doped with cholesterol [6] and glycosphingolipid [17] also inhibit the annihilation of the ripples below the pretransition. These ripples may be metastable as the samples were not annealed for more than a few minutes at these temperatures.

\section{Discussion and conclusions.}

Dimyristoylphosphatidylcholine multilamellar liposomes in excess water exhibit two well-defined ripple configurations when quenched from temperatures between the pre- and main transition in the $\mathrm{P}_{\beta^{\prime}}$ phase. The asymmetric $\Lambda / 2$ phase ripples have wavelengths of $12.0 \mathrm{~nm}$ and an amplitude of less than $6.0 \mathrm{~nm}$. The symmetric $\Lambda$ phase ripples have an 


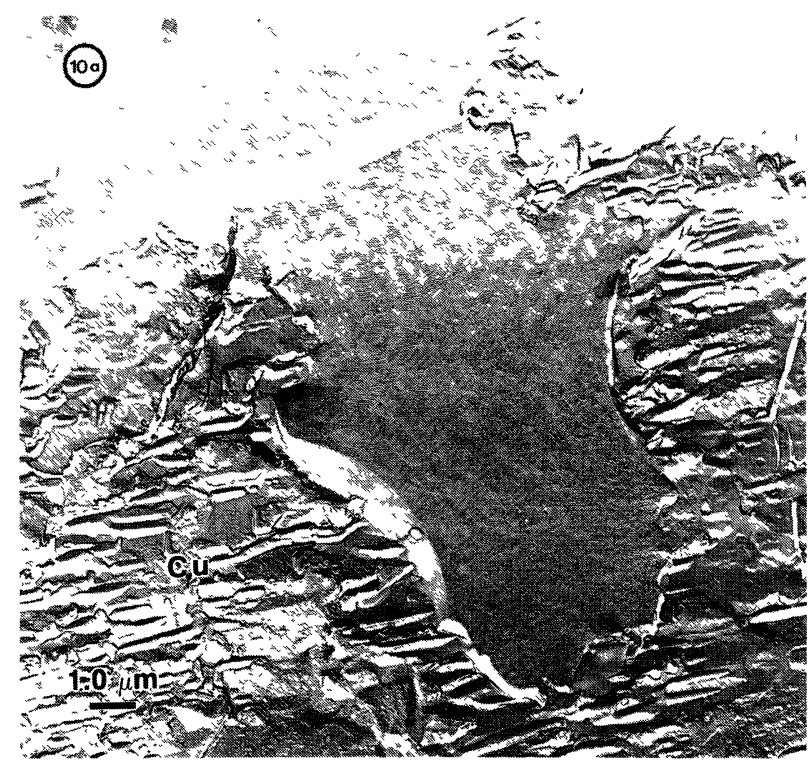

Fig. 10a. - A 5 mole \% DMPE* liposome quenched from a few degrees below the pretransition. A mosaic texture of symmetric, $24.0 \mathrm{~nm}$ ripples remain.

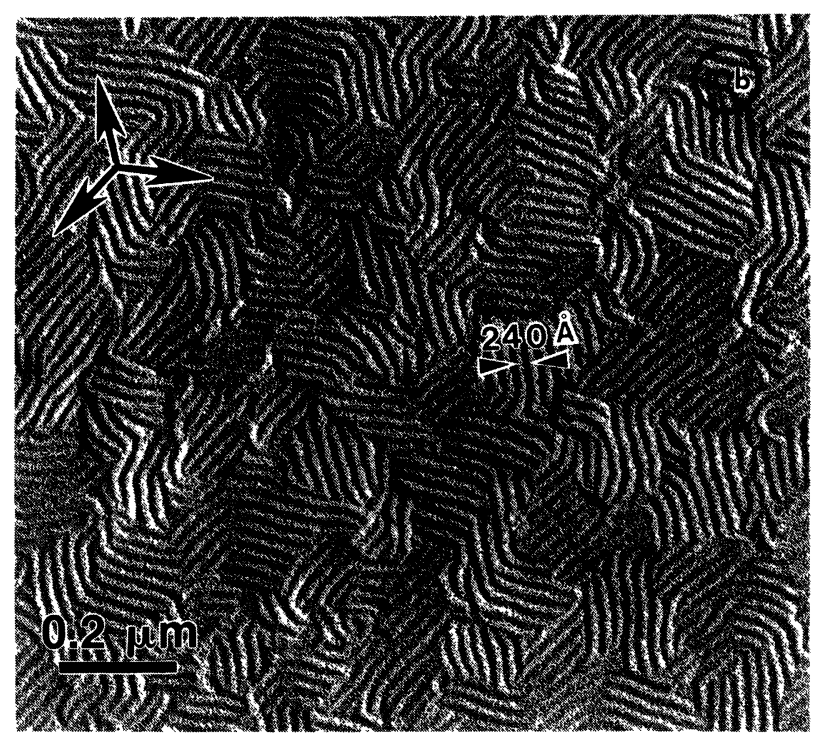

Fig. 10b. - High magnification view of $10 \mathrm{a}$. The threefold symmetry and mosaic texture are clear. DMPE* seems to preserve a hexagonal symmetry in the bilayer. It perhaps prevents the hydrocarbon chains from adopting the flat bilayer and orthorhombic hydrocarbon chain packing of the pure DMPC $\mathrm{L}_{\beta^{\prime}}$ phase.

incommensurate wavelength of $22.0 \mathrm{~nm}$ and an amplitude of at least $9.0 \mathrm{~nm}$. Both ripple phases coexist in pure DMPC liposomes; however, the $\Lambda$ phase is usually confined to smaller diameter, highly curved liposomes. The topologically allowed halfinteger disclinations seem to accommodate the higher curvature better than the integer disclinations allowed in the $\Lambda / 2$ phase. The $\Lambda / 2$ phase is predominant in larger, less curved liposomes ; this curvature dependence of the ripple configurations may explain why the $\Lambda$ phase is not seem in X-ray diffraction experiments on samples of well-aligned DMPC multilayers without excess water.

Addition of N-methyl substituted dimyristoylphosphatidylethanolamine to the DMPC bilayers leads to a disappearance of the pretransition in DSC experiments and both ripple configurations in freeze-fracture micrographs at an extrapolated mole fraction of about 0.15 . The primary effect of the DMPE* is to reduce the average head group size of the lipid molecules in the bilayer; although DMPE* also changes the head group inter- and intra-molecular interactions as is evidenced by a rise of $2-3{ }^{\circ} \mathrm{C}$ in the main transition temperature at 25 mole \% DMPE*. The mismatch between headgroup area and hydrocarbon chain packing volume, believed to be at least partially responsible for the $\mathrm{P}_{\beta^{\prime}}$ phase is eliminated by adding $\mathrm{DMPE}^{*}$, thereby eliminating the need for the $P_{\beta^{\prime}}$ ripples.

Freeze-fracture electron micrographs, however, do not present such a simple picture of the $\mathrm{P}_{\beta^{\prime}}$ phase. Two distinct ripple configurations are observed at all concentrations of DMPE* that showed a pretransition. The wavelength and morphology of the ripples is independent of DMPE* fraction. The ripple directions had three-fold symmetry, reflecting the underlying hexagonal lattice of the hydrocarbon chains. The fraction of $\Lambda$ phase increased with increasing DMPE* fraction, although it is difficult to be quantitative for reasons discussed earlier. The density of disclinations and other defects in the ripple organization also increased with DMPE* fraction. Grain boundaries between different ripple orientations and different ripple configurations also grew more frequent and often intersected at sharp $\left(<120^{\circ}\right)$ angles. In certain regions of 10 mole \% DMPE* liposomes, usually near ripple grain or phase boundaries, the $\Lambda$ phase ripples lost their translational symmetry and became jumbled, although the ripple configurations remained identical. Thus, DMPE* molecules appear to disrupt the underlying hexagonal symmetry, especially in regions of high stress. This loss of translational order may presage the elimination of the $\mathrm{P}_{\beta^{\prime}}$ phase. At and above 25 mole \% DMPE*, liposomes quenched from a couple of degrees below the main transition exhibited flat bilayers without ripples.

Our results are difficult to reconcile with many of the continuum theories of the $\mathrm{P}_{\beta^{\prime}}$ phase $[8,10,11]$. The invariance of the ripple wavelength and configuration with respect to DMPE* fraction suggests that the ripples have a definite number of molecules and their interactions and relative displacement are difficult to change. Most theories predict a continuous, and often large, variation of ripple 
wavelength and amplitude with various parameters describing spontaneous curvature (related to area mismatch between heads and tails), temperature, and ionic strength. X-ray studies have shown that the ripple wavelength is independent of temperature in the presence of excess water [4]. In our study, we presumably varied the spontaneous curvature of the monolayer by equalizing the average areas of the heads and tails. Although the fraction of long wavelength ripples increased with DMPE* fraction, the individual ripple wavelengths and morphologies remained the same. The number of molecules and their relative displacement in the ripple appears to be fixed ; DMPE* acts to lower the energy of and stabilize defects and boundaries between ripples of different orientation or configuration. It seems necessary to include more information about the molecular structure into new models of the $P_{\beta^{\prime}}$ phase [9] to describe the ripple shape and wavelength.

\section{Acknowledgments.}

We would like to acknowledge the help of $\mathrm{M}$. J. Sammon and P. Finn in preparing the freeze-fracture replicas, and D. W. Berreman, S. Meiboom, M. Marcus, and W. Heffner for their patient understanding in setting up the optical diffractometer. We are especially grateful for continuing discussions of the physics of corrugated phases with W. W. Webb, P. E. Cladis, J. Sethna, J. Carlson, and D. Wack. MS acknowledges the support of the National Science Foundation through a Joint Industrial - University grant \#DMR-8404942 to P. E. Cladis and W. W. Webb.

\section{References}

[1] TARdieu, A., Luzzatti, V. and Reman, F. C., J. Mol. Biol. 75 (1973) 711.

[2] ShIPley, G. G. in Biological Membranes, D. Chapman and D.F.H. Wallach, eds., Vol. 2 (Academic Press, New York) 1973, pp. 1-89.

[3] Chapman, D., Q. Rev. Biophys. 8 (1976) 185.

[4] JANiAK, M. J., SMAll, D. M. and Shipley, G. G., J. Biol. Chem. 254 (1979) 6068.

[5] Chapman, D., Ann. NY Acad. Sci. 195 (1972) 195.

[6] Ruppel, D. and Sackmann, E., J. Physique 44 (1983) 1025.

[7] Stamatoff, J., Feuer, B., Guggenheim, H. J., Tellez, G. and Yamane, T., Biophys. J. 38 (1982) 217.

[8] Doniach, S., J. Chem. Phys. 70 (1979) 4587.

[9] Pearce, P. A. and Scott, H. L. Jr., J. Chem. Phys. 77 (1982) 951.

[10] Gebhardt, C., Gruler, H. and Sackmann, E., $Z$. Naturforsch. C 32 (1977) 581.

[11] Falkovitz, M. S., Seul, M., Frisch, H. L. and McConnell, H. M., Proc. Natl. Acad. Sci. 79 (1982) 3918.

[12] Hauser, H., Pascher, I., Pearson, R. H. and Sundell, S., Biochim. Biophys. Acta 650 (1981) 21.

[13] Vaughn, D. J. and KeOugh, K. M., FEBS Lett. 47 (1974) 158.

[14] Yeagle, P. L., Acc. Chem. Res. 11 (1978) 1.

[15] Misell, D. L., Image Analysis, Enhancement, and Interpretation (North-Holland Publishing Co., Amsterdam) 1978, p. 53-70.
[16] Schneider, M. B., Chan, W. K. and WebB, W. W., Biophys. J. 43 (1983) 157.

[17] Tillack, T. W., Wong, M., AllietTA, M. and Thompson, T. E., Biochim. Biophys. Acta 691 (1982) 261.

[18] Krbecek, R., Gebhardt, C., Gruler, G. and Sackmann, E., Biochim. Biophys. Acta 554 (1979) 1. This paper describes a method of analysing freeze-fracture images using densitometry of the negative, which in theory can determine the heights of ripples. However, in practice the procedure is limited by an experimental calibration constant that is difficult to determine unambiguously.

[19] HARRIS, W. F. in Fundamental Aspects of Dislocation Theory, J. A. Simmons, R. de Wit and R. Bullough, Natl. Bur. Stand. (U.S.) Spec. Publ. 317 (1970) 579-582.

[20] Hatta, I., Kato, S. and OhKi, K., Mol. Cryst. Liq. Cryst., in the press.

[21] Silvius, J. R., ReAD, B. D. and McElhaney, R. N., Biochim. Biophys. Acta 555 (1979) 175.

[22] LunA, E. J. and McConnell, H. M., Biochim. Biophys. Acta 466 (1977) 381.

[23] Carlson, J. M., Langer, S. A. and Sethna, J. P., Phys. Rev. Lett., submitted ; CARLSON, J. M. and Sethna, J. P., Phys. Rev. A, submitted.

[24] WaCk, D., Schneider, M. B., WebB, W. W. and ClADIS, P. E., Biophys. J. in preparation. 